\title{
Philosophiques
}

\section{Le sublime et la fiction}

\section{Peter McCormick}

Volume 23, numéro 1, printemps 1996

Critères esthétiques et métamorphoses du beau

URI : https://id.erudit.org/iderudit/027369ar

DOI : https://doi.org/10.7202/027369ar

Aller au sommaire du numéro

Éditeur(s)

Société de philosophie du Québec

ISSN

0316-2923 (imprimé)

1492-1391 (numérique)

Découvrir la revue

\section{Citer cet article}

McCormick, P. (1996). Le sublime et la fiction. Philosophiques, 23(1), 93-112. https://doi.org/10.7202/027369ar

\section{Résumé de l'article}

Il y a des oeuvres d'art poétiques, et en particulier quelques poèmes modernistes, qui nous offrent des représentations du sublime dans un de ses aspects les plus problématiques. Cet aspect comporte la simultanéité des éléments à la fois subjectifs et objectifs. Mais comment peut-on comprendre le sublime sous ce double masque ? Comment se fait-il que le sublime puis- se être à la/ois objectif et subjectif? Dans cet article je propose d'articuler ces deux aspects du sublime en ayant recours aux réflexions tardives de Kant dans son Opus postumum sur le Selbstsetzungs- lehre, sur ce que j'appellerai « une auto-position fictive ".
Ce document est protégé par la loi sur le droit d'auteur. L'utilisation des services d'Érudit (y compris la reproduction) est assujettie à sa politique d'utilisation que vous pouvez consulter en ligne.

https://apropos.erudit.org/fr/usagers/politique-dutilisation/ 


\title{
IE SUBLIME EI LA FICIION
}

\author{
PAR \\ Peter McLormick
}

\begin{abstract}
RÉSUMÉ: Il y a des œuvres d'art poétiques, et en particulier quelques poèmes modernistes, qui nous offrent des représentations du sublime dans un de ses aspects les plus problématiques. Cet aspect comporte la simultanéité des éléments à la fois subjectifs et objectifs. Mais comment peut-on comprendre le sublime sous ce double masque ? Comment se fait-il que le sublime puisse ètre à la fois objectif et subjectif?

Dans cet article je propose d'articuler ces dewx aspects du sublime en ayant recours aux réflexions tardives de Kant dans son Opus postumum sur le Selbstsetzungslehre, sur ce que j'appellerai " une auto-position fictive".
\end{abstract}

ABSTRACT : Some poetic works of art, and in particular some modernist poems, present us literary representations of the sublime in one of its most searching, and still problematic, aspects. This aspect of the sublime, unlike many others, involves the simultaneity of both subjective and objective elements.

But how are we to understand the sublime in such a double guise? How can the sublime be simultaneously both objective and subjective?

My suggestion here is that we can best articulate both the objective and subjective aspects by making use of Kant's late reflections in the Opus Postumum on the Selbstsetzungslehre, that is, on what $I$ will call $a$ fictional self-positing.

La philosophie transcendantale précède l'affurmation des choses pensées, comme leur prototype, dans lequel elles dowent être placées.

Kant

Écoutons tout d'abord l'une des voix les plus vibrantes de la poésie moderne afin de nous plonger dans une atmosphère propice à mieux saisir, alors que la modernité touche à sa fin, la notion de sublime. Avec ses réminiscences de Mallarmé et Baudelaire, Eliot, Rilke et Valéry, Dante et Leopardi, et sa perpétuelle obsession de la souffrance, tant concrète qu'abstraite, la voix d'Eugenio Montale continue de résonner dans cet extrait d'Os de seiche, publié en 1925 : 
Tu demandes si tout ainsi s'efface dans cette mince brume de souvenirs : si dans l'heure qui somnole ou si dans le soupir du récif s'accomplit tout destin.

Je voudrais te dire non, et qu'approche

l'heure où tu passeras sur l'autre bord du temps :

peut-être seul qui le veut s'éternise,

et cela, tu le pourras - qui sait - non moi.

Pour la plupart, je pense, il n'est point de salut, mais tel bouleverse tous les plans.

franchit la passe, tel qu'il se voulait se retrouve.

Avant de m'effacer je voudrais t'indiquer

cette voie d'évasion

instable comme dans les champs houleux

de la mer la ride ou l'écume.

Je te donne aussi mon avide esperance.

Pour des jours neufs, lassé, je ne sais la nourrir :

Je l'offre en gage à ton destin, pour qu'il t'épargne ${ }^{1}$.

Nombreux sont ceux qui, de nos jours, se sentent mal à l'aise, voire mème irrités ou révulsés face à un tel poème. Car, vivant dans une époque postmoderne, nous éprouvons une certaine difficulté à accepter ce qui nous semble n'ètre que le produit d'une certaine affectation littéraire où se mêlent une simplicité trompeuse, les artifices d'une naïveté feinte, l'évocation douteuse d'une réalité transcendant les limites de notre monde matériel, la glorification d'une souffrance somme toute forcée et le style pompeux d'un dialogue imaginaire.

Une telle affectation littéraire ne reflète-t-elle autre chose que l'influence qu'a eue, sur la poésie lyrique de Montale, la Ligurie de sa jeunesse ? Comment pourrions-nous dès lors, raisonnablement, faire nôtre ce poème ? Mais surtout, en ces temps de crise et à la fin $\mathrm{du}$ siècle le plus sanglant de notre histoire, une telle question a-t-elle encore une raison d'ètre?

Je me propose ici de montrer en quoi ce poème, si marqué qu'il soit par le modernisme, continue aujourd'hui encore, comme tant d'autres poèmes, de nous donner à penser. Car il nous présente le sublime sous l'un de ses aspects les plus ardus et, somme toute, les plus problématiques : celui de son caractère à la fois subjectif et objectif. Afin d'expliciter et de résoudre les tensions conceptuelles résultant de ce caractère double du sublime et, plus particulièrement, de comprendre son aspect subjectif, il me semble utile de se reporter aux écrits posthumes de Kant sur ce que j'appellerai "l'autoposition fictive".

\section{La représentation du sublime}

Montale publia Os de seiche en 1925 peu après qu'eut paru, dans In Baretti, un de ses essais les plus remarqués et qu'il eut contresigné la réponse adressée par Benedetto Croce au Manifeste des

1. Extrait de * La maison sur la mer * d'Eugenio Montale, Os de seiche, traduit par Patrice Angelini avec le concours de Louise Herlin et Georges Brazzola, Paris, NRF, Gallimard, 1966, p. 189 et 191. 
intellectuels fascistes. Ce recueil de poèmes lyriques composé avec soin et où se succèdent cycles et pièces isolées, telles que " La maison sur la mer *dont nous venons de citer un extrait dans l'introduction, semble renvoyer, par le biais de somptueuses évocations lyriques, aux paysages des cōtes liguriennes où Montale passa, dans sa jeunesse, ses vacances à Monterosso au sud de Gènes.

Mais cette recherche d'un art figuratif distinctif n'était pas sans ambiguité. Prenant ses distances vis-à-vis du style ampoulé de D'Annunzio, Montale cherche à retrouver les rythmes forts et âpres de Dante, la rime aspre de "L'Enfer * et un mouvement plus ancien, celui de la rime petrose des "Rimes pour la "Dame Pierre " et commence, avec Os de seiche, une exploration critique du modernisme et de sa postérité qu'il continuera de mener tout au long de son œuvre. Cette exploration s'articulera principalement autour de la relation tendue, devenue l'objet de violentes controverses dans notre époque postmoderne, qui existe entre poésie et vécu, entre poésie et philosophie. Les plus beaux poèmes de Montale sont ceux où, plus qu'ailleurs, i1 cherche à appréhender ce sentiment profond et ténébreux, parfois accablant, d'une souffrance abstraite et immense, celui du pathos abyssal des choses.

Bien que partageant les convictions antifascistes de Croce quant à la séparation de l'art et de la politique, Montale se démarque des propos alarmistes et parfois prophétiques de celui-ci sur le modernisme en tant que nationalisme culturel favorisant l'avènement du fascisme. Cherchant à se dégager de l'emprise du régionalisme de la poésie ligurienne qui caractérisera son ami Camillo Sbarbaro, Montale, abandonnant les paysages marins de la Ligurie pour Florence, "terre ferme des idées, de la tradition et de l'humanisme" s'intéresse surtout au caractère cosmopolite et européen du modernisme.

C'est cette approche élargie du modernisme, que l'on pressent dans la poésie et les essais de Montale tout autant que dans son intérêt pour l'avant-garde de son époque - les préludes pour piano de Debussy, les romans de Svevo, les esquisses de Morandi et les pièces de Pirandello - que je souhaite prendre comme point de départ pour ces quelques réflexions sur la fiction, la poésie, et le sublime. Il semble en effet que le climat culturel dans lequel nous vivons aujourd'hui, que ce soit en Amérique du Nord, en Europe ou au Japon, se prète tout particulièrement, et aussi surprenant que cela puisse paraitre au premier abord, à la réexamination critique d'une conception du sublime qui, bien que considérée par beaucoup comme dépassée, peut nous offrir les moyens conceptuels d'appréhender les relations complexes qui unissent l'art, la vie et la raison.

De nouvelles perspectives sur le modernisme et le postmodernisme ouvertes par des recherches récentes en histoire et en sociologie nous ont fait comprendre à quel point notre époque demeurait marquèe, tant au niveau culturel que social ou historique, par les grands moments artistiques du modernisme. Car c'est sur un arrière-

2. Tiré de Intenzioni (Intervista imaginaria), 1946, cité par W. Arrowsmith dans son introduction à sa traduction d'Osse di Sepia:Cuttlefish Bones. New York, Norton, 1993, p. xvi. 
plan de modernisme que se détache ce discours artistique et critique postmoderne qui occupe actuellement l'avant-scène. Mais nous hésitons, aujourd'hui encore, à donner d'une œuvre d'art une interprétation qui risque d'être trop figurative ou trop réaliste: nous nous refusons à chercher dans notre vie et dans celle des sociétés qui nous entourent les moyens de comprendre la poésie de Montale et ses élans vers le sublime, tels qu'ils se présentent dans Os de seiche, de peur de sombrer dans une forme douteuse d'esthétisme naif ou de moralisme réaliste.

Guel que soit le véritable propos d'œuvres telles que Os de seiche, et malgré l'évident effacement de la frontière entre physique et métaphysique qui s'y lit - "la passe ", "cette voie d'évasion/instable comme les champs houleux/de la mer la ride ou l'écume" - nous préférons feindre de croire, en conformité avec les interprétations instrumentales, relativistes et réflexives du postmodernisme, que de telles cuvres possèdent une dimension propre qui nous est inaccessible.

La poésie, a-t-on entendu dire, ne saurait, après Auschwitz, donner de leçon à personne. La poésie ne saurait nous inviter ou nous inciter à une quelconque réflexion. Il lui faut, nous dit-on, se contenter de briller, en quelques occasions, des feux scintillants de sa propre perfection et, dans son propre équilibre paisible, réfléchir sans fin sa propre beauté.

Car parler de la poésie de Montale, ou de toute autre œuvre d'art. en d'autres termes que ceux qui leur sont propres serait violer le préjugé postmoderne qui veut que littêrature, d'une part, et politique ou morale, d'autre part, soient séparées par une frontière infranchissable que Montale et Croce ont eux-mêmes contribué à établir. Nous ne ferions, ce faisant, que retomber dans l'hérésie conceptuelle de la représentation mimétique qui, depuis Aristote, veut que la poésie, ou toute autre forme d'art, soit plus philosophique que l'histoire.

Dans une telle perspective, peu populaire de nos jours, une certaine forme de poésie semblerait pouvoir, par l'évocation de certaines situations réelles, rendre manifeste une certaine forme d'universaux. Une telle évocation semblerait, de plus, et selon une guise à laquelle nous ne semblons plus aujourd'hui guère nous intéresser, se référer à une dimension qui la transcende et toucher, parfois même, à la morale en tant que telle. Ne se contentant pas d'être le simple instrument d'un plaisir fugitif et personnel, une telle forme de poésie, par l'évocation d'une certaine forme d'actes et de discours, serait à mème d'inviter le lecteur, ou l'exégète, à une réflexion qui lui permettrait, dans une perspective imaginative, cognitive et sociale, d'envisager divers idéaux moraux et leur impact potentiel sur son esprit.

Une telle approche de la représentation mimétique qui, défendant l'idée que la poésie puisse renvoyer à une réalité transcendant la simple représentation, à des auteurs réels et non fictifs ainsi qu'à une signification précise, stable et dëfinie et, dès lors, permettre une critique qui, normative et non simplement descriptive, soit à même d'évoquer le sublime et le pathos véritables, est, de nos jours, trop souvent négligée, voire même dénigrée. Telle est l'approche si souvent, et si vigoureusement, critiquée par bon nombre d'ouvrages 
récents qui, se réclamant d'un certain formalisme linguistique, d'un certain post-structuralisme, d'une certaine déconstruction, d'une certaine critique sociale ou d'un certain féminisme, cherchent à s'imposer en tant que nouvelle norme postmoderne de la critique littéraire.

Mais, quels que soient par ailleurs les mérites non négligeables de ces différentes approches - et ceux-ci sont nombreux bien qu'il semble, aujourd'hui plus que jamais, et au vu des différents scandales qui ont touché la déconstruction et ceux qui s'en réclament, nécessaire d'en faire un examen minutieux et approfondi - je pense que n'avons pas encore épuisé les possibilités que nous offre une approche mimétique de la littérature. Je pense également que nous négligeons trop souvent, en ces temps de troubles historiques, culturels et sociaux, la raison pour laquelle des poèmes, tels que ceux de Montale dans Os de seiche, peuvent encore nous toucher aujourd'hui et ce, alors que nous essayons, non sans difficulté, de définir la relation ambiguë que nous entretenons avec la poésie de la souffrance.

C'est dans un tel contexte que je m'intéresse tout particulièrement, dans la poésie moderne de Montale, au thème du varco la passe, à la fois dans le sens de passage et d'ouverture - où se manifestent hésitation et ambivalence quant à une possible transcendance alors même que la primauté d'une immanence des plus intenses y est délibérément traduite par une poésie où se reflètent appauvrissement, vacuité et anéantissement de soi. Il me semble nécessaire, afin de comprendre une telle poésie de la souffrance abstraite, de se replonger, toujours et encore, dans les réflexions de Kant sur le sublime qui, malgré leur complexité, continuent de nous offrir une perpective unique sur le " sujet " ambigu de l'interprétation.

\section{Le sublime kantien en poésie moderne}

Le poème Maison sur la mer de Montale essaye, à sa façon, de résoudre l'inévitable dilemme du destin et de la fatalité. Ce poème nous propose d'envisager, délaissant nos convictions les plus établies, ce qui ne peut être, comme le poète lui-même le reconnaît, qu'un vague espoir pour une infime minorité. Il s'agit ici de trouver un varco, un passage, entre deux pôles fondamentaux et, ce faisant, de pouvoir dépasser cette situation de polarité fondamentale dont souffre tout ètre humain.

Cette polarité fondamentale est celle que nous ressentons ici dans la tension existant entre le silence temporel, fini, riche en couleurs mais finalement mortel d'une stricte immanence représentée par la terre et lappel transcendant, intemporel, infini et toujours imprévisible d'une éternelle aspiration que représente la mer. S'il nous faut, d'après le poète se contenter de cultiver paisiblement la terre par l'imagination, son interlocutrice fictive semble nous inviter à prendre le risque, ne serait-ce qu'une fois, d'explorer les périls de la mer). Mais le poème, en tant que tel, ne traite pas tant de cette polarité que de la façon de surmonter la souffrance que cause irrémédiablement en nous cette perpétuelle polarité apparemment irréductible, cette souffrance immense qui s'abreuve à la source intarissable et insondable du pathos des choses. Comment dès lors 
s'attaquer à ce nœud gordien où s'entremēlent le subjectif et l'objectif?

Dans l'un de ses essais, Montale écrit : "Le “ moi " transcendantal est un fanal qui n'illumine qu'une infime portion de l'espace qui s'offre à nous, un fanal qui nous guide vers une condition qui, étant impersonnelle, n'est plus humaine. "Plutōt que de rechercher à savoir quelle aurait pu ètre l'influence de Kant sur Montale ou sur d'autres poètes de la souffrance qui lui sont contemporains, je préfère ici prendre comme point de départ cette réflexion de Montale afin de montrer comment, aussi surprenant que cela puisse paraitre, les écrits de Kant sur le sublime peuvent, à travers les différents usages que celui-ci fit du terme " transcendantal "dans sa quête d'une "philosophie transcendantale ", se révéler utile à résoudre le problème qui nous préoccupe ici.

Kant, après plusieurs approximations, définit la philosophie transcendantale comme le fondement déterminant de la raison. "La philosophie transcendantale ", écrit-il de façon synthétique dans un passage de l'Opus postumum rédigé vers 1801, "est le principe général déterminant la raison en raisons théorico-spéculative et éthico-pratique ${ }^{3}$. Une telle détermination, poursuit-il, constitue "l'unitê du tout conditionné comme totalité (universum) des choses dans leur unité synthétique selon les concepts a priori des éléments de celle-ci : Dieu, le monde et l'homme assujetti dans le monde à la loi morale ". Mais en quoi cette dernière formulation de la philosophie transcendantale diffère-t-elle des interprétations que Kant en avait donné auparavant et comment peut-elle nous permettre de mieux comprendre en quoi consiste le contexte, offert par la poésie moderne, de la représentation du sublime en tant que transcendance de l'objectif et du subjectif?

La formulation que donne Kant de la philosophie transcendantale dans l'Opus postumum diffère en de nombreux points de celle qu'il en donnait, par exemple, dans la Critique de la raison pure. Kant pensait alors que la philosophie "transcendantale ", en tant que critique de la raison pure, pourrait définir la raison hors du domaine empirique. Cette nouvelle formulation de la critique devait nous permettre d'appréhender une forme de " connaissance qui s'occupe en général non pas tant d'objets que de notre mode de connaissance des objets mais avec nos concepts a priori des objets ${ }^{5}$ ".

Ce sont de tels objets indéterminés qui constituent de façon générale pour la pensée les objets intentionnels appréhendés par la faculté de juger. C'est ainsi que Kant, dans l'Opus postumum, écrit que la philosophie transcendantale "appréhende de façon immédiate les objets (ens summum, summa intelligentia, etc.) qui, indépendamment

3. Kant, Opus postumum, dans Kant's Gesammelte Schriften, herausgegeben von der Preußischen Akademie der Wissenschaften, Band XX1, Berlin, Walter de Gruyter \& Co, 1938, p. 79-80. Traduit par P. McCormick \& L. Perrin.

Toutes les références à l'Opus postumum, quelle que soit la traduction utilisée, seront données sous la forme suivante : (AK XXI, 79-80).

4. Ibid., (AK XXI, 80).

5. Kant, Critique de la raison pure, traduit par J.-L. Delamarre et F. Marty à partir de la traduction de J. Barni, Paris, Gallimard, 1980, p. 83 (AK IV, 8 - A $11-12 /$ B 25). 
de toute expérience, sont postulès par la raison pure comme objets [nécessaires] à la possibilité de [son] expérience ${ }^{6}$ * La philosophie transcendantale ne constitue donc pour Kant qu'un moyen d'explorer la façon dont la notion d'objet en général peut mener à la notion d'objet en tant que concept de la connaissance a priori?

Kant avait néanmoins développé entre temps, dans les Prolégomènes à toute métaphysique future qui pourra se présenter comme science, une différente interprétation de la philosophie transcendantale. Résistant à la tentation de renoncer à l'appellation de "philosophie transcendantale "pour celle d' idéalisme critique", Kant préféra y redéfinir ce que devait ètre la "philosophie transcendantale ". Il substitua ainsi l'expression " expériences possibles "à celle d' "objets en général ".

Il en résulte que la philosophie transcendantale, d'exploration de " concepts a priori d'objets en général " qu'elle était, devient exploration "des conditions a priori d'une expérience possible ". Comme Kant l'écrit : " [...] le mot "transcendantal " [...] ne signifie pas quelque chose qui s'élève au-dessus de toute expérience, mais ce qui certes la précède (a priori) sans ètre destiné cependant à autre chose qu'à rendre possible uniquement une connaissance empirique ${ }^{8}$ ". La raison est donc ici subordonnée à une nouvelle définition de la tâche originelle de la philosophie transcendantale.

Mais la définition donnée dans les Prolégomènes n'est pas définitive. Car Kant comprit vite quayant modifiè la définition de la philosophie transcendantale, il lui fallait modifier celle de la métaphysique en conséquence. Kant comprit en particulier que ses Premiers principes métaphysiques de la science de la nature ne pouvaient à la fois fournir un locus a priori permettant de classifier de façon systématique les forces particulières, telles que l'attraction ou la répulsion, qui se présentent de façon empirique dans la nature et les raisons a priori "d'espérer que la nature permet une telle classification $^{9}$."

Si Kant, en réponse à Hume, avait déjà démontré que l'expérience empirique ne pouvait garantir la nécessité, il prenait dès lors conscience que l'expérience ne pouvait pas non plus garantir la systématisation. Il lui fallait donc découvrir quelque principe encore ignoré afin de comprendre comment la nature elle-mème pouvait permettre une telle classification. C'est ainsi que Kant écrit, dans sa première introduction à la Critique de la faculté de juger, "la faculté de juger réfléchissante ne pourrait entreprendre, suivant sa propre nature, de classifier la nature tout entière d'après ses diversités

6. Kant, Opus postumum, dans Kant's Gesammelte Schriften herausgegeben von der Preußischen Akademie der Wissenschaften, op. cit., (AK, XXI, 80).

7. $C f$. Foerster, "Introduction " à l'Opus postumum traduit par M. Rosen et lui-mème, Cambridge, Cambridge University Press, 1993, p. xxx.

8. Kant, Prolégomènes à toute métaphysique future, Appendice, traduit par $\mathrm{J}$. Rivelaygue, dans CEures philosophiques, vol. 2, Paris, Gallimard, 1985, p. 161 (AK IV, 373).

9. E. Foerster, "Introduction "à l'Opus postumum, op. cit., p. xxxiv. 
empiriques, si elle ne présupposait pas que la nature spécifie ellemême ses lois transcendantales d'après quelques principes ${ }^{10}$ ".

C'est un tel principe que Kant définit dans sa Critique de la faculté de juger comme ètant la finalité de la nature, l'idée que les lois universelles de la nature peuvent être considérées comme étant empiriquement déterminées par la structure de la connaissance humaine. C'est ainsi que Kant écrit: "La beauté naturelle autonome nous révèle une technique de la nature qui la représente comme un système obéissant à des lois dont le principe échappe à l'ensemble de notre entendement; il s'agit du principe d'une finalité qui se rapporte à l'usage de la faculté de juger en présence de phénomènes ${ }^{11}$ ". Ce qui ne semblait que contingence et hasard dans la nature peut maintenant ètre considéré comme étant à la fois nécessité et systématisation. Encore fallait-il définir le lien unissant une comprèhension générale des sciences aux sciences en tant que telles.

Kant reprit en 1796 un travail qu'il avait commencé près d'une décennie auparavant et qui constitue un "passage "des Premiers principes métaphysiques de la science de la nature à ce qu'il appelait les forces réelles de la nature en physique. Ce travail se caractérise par le fait que Kant y délaisse l'examen des forces motrices (l'attraction et la répulsion sont les exemples donnés par Kant dans l'Opus postumum ${ }^{12}$ ) pour celui de la conscience qu'a le sujet pensant de ses propres forces motrices ou dymamiques. Kant écrit ainsi dans l'Opus postumum : "Le sujet pensant se crée un monde comme sujet d'une expérience possible dans l'espace et le temps. Cet objet n'est qu'un seul monde. - En ce monde se posent des forces motrices $[\ldots]^{13}$ ". L'étude du sujet dynamique, que Kant intitule "Selbstsetzungslehre" - théorie de l'autoposition de soi - constitue l'un des principaux thèmes des derniers écrits de l'Opus postumum. Et c'est dans ce contexte particulier que Kant propose une dernière définition de la philosophie transcendantale formulée en termes négatifs et positifs.

Définie de façon négative, la philosophie transcendantale consiste en l'exploration de la possibilité d'une connaissance synthétique a priori fondée non sur l'intuition mais sur des concepts. Définie de façon positive, elle est "l'acte de conscience par lequel le sujet devient son propre auteur et, ce faisant, constitue le tout de l'objet des raisons technico-pratique et éthico-pratique en un système ${ }^{14}$ ". En résumé, la philosophie transcendantale "devient la théorie de l'autoposition de soi, de l'autoconstitution de la raison à la lumière des trois idées ou images originelles et nécessaires qui lui donne matière à [former] un savoir synthétique à partir des concepts : "Je dois avoir des objets de ma pensée et les appréhender, car autrement

10. Kant, "Première introduction "à la Critique de la faculté de juger, trad. par A. J.-L. Delamarre, dans CEuvres philosophiques, vol. 2, op. cit., p. 868 (AK $\mathrm{XX}$, p. 215), cité par E. Foerster dans son "Introduction" à l'Opus postumum op. cit., p. xxodiv.

11. Kant, Critique de la faculté de juger, trad. par J.-R. Ladmiral, M. B. de Launay et J.-M. Vaysse, dans CEures philosophiques, vol. 2, op. cit.. p. 1011 (AK V, 246).

12. Kant, Opus postumum, textes choisis et trad. par J. Gibelin, Paris, Librairie philosophique J. Vrin, 1950, p. 14. (AK XXI, 24).

13. Ibid., idem (AK XX1, 23).

14. Ibid., p. 34 (AK XX1, 78). Trad. complètée par P. McCormick et L. Perrin. 
je suis inconscient de moi-mème ${ }^{15}$ ", écrit Kant. La raison (ou esprit de l'homme) crée donc inévitablement ces idées (Dieu, le monde, le devoir) afin de s'autopositionner, de devenir consciente de soi en tant qu'ètre naturel et que personne ${ }^{16}$.

Kant, à la fin de sa vie, écrit : "La philosophie transcendantale précède l'affirmation des choses pensées, comme leur prototype, dans lequel elles doivent être placées ${ }^{17}$ ". La raison, prenant ici la forme ultime de l'esprit humain, doit assumer la double nécessité de se créer et de s'appréhender soi-mème tout en créant et en appréhendant des idées telles que celle de Dieu, du monde et du devoir.

L'évolution que connut chez Kant la philosophie transcendantale demeure, mème si on la simplifie à l'extrème comme j'ai dū le faire ici, à la fois complexe et obscure. Nombreux sont les points qui nécessiteraient un examen et une analyse plus poussés. Il n'en demeure pas moins qu'on y discerne les grandes lignes de ce qui constitue une réflexion extraordinaire, et même révolutionnaire, sur ce pôle positif de la philosophie transcendantale qu'est la dimension créatrice de la raison. Cette réflexion, par son caractère original et novateur, sera reprise par Schelling, Fichte et Hegel dans ce qui constituera le temps fort de l'Idéalisme allemand et, par l'intermédiaire de Coleridge, qui passa une année en Allemagne à la fin des années 1790, le point de départ théorique, en Angleterre, du Romantisme. La critique tardive que Kant fera en retour de l'Idéalisme allemand et du Romantisme anglais sera à son tour reprise par le modernisme qui émergera, dans le domaine des arts, à la fin du dixneuvième siècle. Il semble donc intéressant d'étudier ce qui, à travers l'évolution que connait la philosophie transcendantale dans la pensée kantienne, constituera ce que Kant définira, sur sa fin, comme le caractère créatif de la raison autonome.

\section{Fiction, raison et esprit}

Dans la Critique de la faculté de juger Kant distingue les idées rationnelles des idées esthétiques ${ }^{18}$. Une idée rationnelle se rapporte à un concept par un principe objectif tandis qu'une idée esthétique se rapporte à une intuition par un principe subjectif. Le principe subjectif par lequel une idée esthétique se rapporte à une intuition relève plus particulièrement de l'" accord réciproque des facultés de connaître (de l'imagination et de l'entendement) ". Aucune idèe ne peut garantir la connaissance (Erkenntnis, c'est-à-dire le produit du processus d'acquisition du savoir [Wissen] $)^{20}$. Car une idée rationnelle "contient un concept (du suprasensible) auquel on ne peut jamais donner une intuition qui lui soit conforme "tandis qu'une

15. Kant, Opus postumum, trad. par F. Marty, Paris, PUF, 1986, p. 352 , n. 516, (AK XXI, 82).

16. E. Foerster, "Introduction "à l'Opus postumum, op. cit., p. xliv.

17. Kant, Opus postumum op. cit., p. 263 (AK XXI, 7), cité par E. Foerster dans son "Introduction "à l'Opus postumum op. cit., p. xliv.

18. Kant, Critique de la faculté de juger, op. cit., p. 1131-2 (AK V, 342).

19. Ibid., idem.

20. Ibid., p. 1286-7 (AK V, 475).

21. Ibid., P. 1132 (AK V, 342). 
idée esthétique " est une intuition (de l'imagination) pour qui on ne peut jamais trouver un concept adéquat ${ }^{22}$ ".

Kant considère qu'une idêe rationnelle est un " concept indémontrable de la raison "23 "tandis qu'une idée esthétique est une "représentation inexponible de l'imagination ${ }^{24}$. Les idées esthétiques ne peuvent être expliquées du fait que l'entendement est incapable de saisir par ses concepts particuliers la totalité de l'intuition imaginative dans son libre jeu. Les idées esthétiques, si elles ne peuvent être conceptualisées, peuvent néanmoins être représentées. Elles ont de plus une utilité et une nécessité toutes particulières : elles servent de principes régulateurs. Elles délimitent ainsi la sphère possible de l'entendement et marquent la frontière, pourrions-nous dire, entre les vivants et les morts.

Kant en donne une description plus détaillée lorsqu'il aborde le géni $^{25}$ et identifie la faculté particulière de représenter les idées esthétiques comme l'" esprit ${ }^{26}$, "principe qui insuffle sa vie à l'esprit "27. L'" esprit "est donc ici précisément ce qui produit les idées esthétiques sous la forme de représentations de l'imagination qui, Kant ajoute-t-il, "donne[nt] beaucoup à penser, sans pourtant qu'aucune pensée déterminée, c'est-à-dire aucun concept ne puisse lui ètre approprié et, par conséquent, qu'aucun langage ne peut exprimer complètement ni rendre intelligible ${ }^{28}$ ".

Kant avance alors que l'" esprit " est tout particulièrement visible dans certaines poésies. Les expériences de la vie de tous les jours, telles que la vie ou la mort, de même que des idées rationnelles, telles que l'éternité ou l'immortalité sont, dans la poésie, présentées d'une façon qui transcende l'expérience, c'est-à-dire "à un niveau de perfection sans exemple dans la nature ${ }^{29}$ ". (Montale ne parle-t-il pas de la mort, de l'éternité et de l'immortalité lorsqu'il parle du varco comme d'un passage "sur l'autre bord du temps " ?) Kant pense que le poète élabore de telles expressions grâce à une forme particulière d'activité imaginative et créatrice qui " rivalise avec le prélude de la raison dans la recherche d'un idèal suprême ${ }^{30}$ ".

Kant donne un sens particulier au terme de "créatrice "qu'il attribue à cette forme extraordinaire d'imagination. Les représentations intuitives produites par l'imagination du poète lui permettent de penser plus "que ce qui peut y ètre appréhendé et expliqué ${ }^{31}$ ", plus "qu'on ne peut exprimer dans un concept défini par des $\operatorname{mots}^{32}$. Dans de telles représentations imaginatives, les idées esthétiques " anim[ent] l'esprit en lui ouvrant une perspective à perte de vue dans le domaine des représentations apparentées ${ }^{33}$. Ces idées

22. Ibid, idem

23. Ibid., idem.

24. Ibid., idem.

25. Ibid., p. 1096-1103 (AK V, 313-319)

26. Ici comme par la suite, " esprit " traduit mind, c'est-à-dire âme, tandis que esprit traduit spirit (Note du traducteur).

27. Ibid., p. 1097 (AK V, 313).

28. Ibid., p. 1097 (AK V, 314).

29. Ibid., p. 1098 (AK V, 314).

30. Ibid., idem

31. Ibid., P. 1098 (AK V, 315).

32. Ibid., p. 1099 (AK V, 315).

33. Ibid., idem 
émergent des attributs esthétiques variès de lobjet. Des attributs esthétiques qui, écrit Kant, "donnent à l'imagination un élan qui lui permet de penser davantage, quoique sans pouvoir développer explicitement cette pensée, qu'on ne peut comprendre grāce à un concept $^{34}$ ".

De façon plus générale, une idèe esthètique, écrit Kant, " est une représentation de l'imagination, associée à un concept donné, et qui est liée à une telle diversité de représentations secondaires [les attributs esthétiques] dans le libre usage de celles-ci qu'on ne peut trouver pour elle aucune expression qui définisse un concept déterminé ; elle permet de penser, associèe à un concept, bien des choses indicibles dont le sentiment anime les facultés de connaissance, et insuffle un "esprit " au langage considéré comme simple système de lettres ${ }^{35}$ ".

Bien que de nombreux points de cette extravagante doctrine kantienne méritent d'être examinés au vu de ce que sont aujourd'hui les théories, certes controversees, se rapportant au corps, au cerveau et à l'esprit, celles-ci renferment néanmoins un certain nombre d'éléments qui peuvent nous permettre de mieux comprendre ce que peut ètre le caractère à la fois subjectif et objectif du sublime. Car elle a en effet le mérite de définir à la fois la contingence radicale de l'esprit et la capacité qu'a celui-ci d'appréhender ce que Kant appelle la réalitè suprasensible.

Les représentations imaginatives d'idées esthétiques et de leurs attributs présentes dans certains poèmes (prenons ici pour exemple la poésie de la souffrance de notre fin de siècle plutôt que celui, problématique pour Kant, des vers laborieux que Frédéric le Grand écrivit en français) frappent l'esprit tout comme le fait initialement l'incommensurablement grand (le sublime mathématique kantien) ou l'incommensurablement puissant (le sublime dynamique kantien). Car l'esprit ne peut s'empēcher, lorsqu'il fait la douloureuse expérience de la limite qui s'impose à son inexorable élan, de vaciller tout comme notre vision oscille lorsqu'elle est confrontée à une œuvre d'Op Art. Cette oscillation, ce vacillement, révèle à l'esprit l'irrémédiable impossibilité pour lui de conceptualiser l'incommensurable souffrance dont témoignent certains poèmes et l'incommensurable puissance qui déchaine une telle souffrance. L'esprit néanmoins, prenant conscience de cet èlan qui le pousse à vouloir exprimer par les mots ce qui est indicible et ne peut être qu'évoqué, découvre l'un des désirs de la raison. Et dans son combat pour satisfaire, au-delà des limites de l'expérience, ce désir de la raison qui est de conceptualiser cette incommensurable souffrance et la puissance, tout aussi incommensurable, qui est source de cette souffrance, l'esprit découvre en lui ce vacillement incessant et à jamais inassouvi qui est la marque de l'" esprit " humain.

Si ce désir d'appréhender l'expérience du sublime en sa négativité est, comme je viens de l'écrire, un désir de la raison, il ne l'est pas au sens où l'entend Paul de Man lorsqu'il interprète, d'une façon plus que douteuse, celui-ci comme élan vers ce qui peut ètre source de plaisir. Le désir kantien est ici au contraire une faculté de 
l'" esprit " qui " par ses représentations cause de l'effectivité des objets de ces représentations ${ }^{36}$ ". Et ce désir d'appréhender ce que dévoile un tel poème est la marque d'une inéluctable pulsion qui pousse l'" esprit " à vouloir comprendre, de façon rationnelle, ce qui ne peut se trouver, et pour autant qu'une telle chose existe, quaudelà des frontières de l'esprit. Mais un tel désir d'appréhender ce que dévoile ce poème ou, plus généralement, toute poésie de la souffrance humaine, est, avant toute chose, la marque d'un "esprit "qui ne saurait être interprété de façon religieuse. Cette marque de l' esprit * n'est, au contraire, que le signe animateur, bien que vacillant, de cette plénitude du tout qui, de façon rationnelle, ne peut se trouver, et pour autant qu'une telle chose existe, qu'au-delà des frontières de l'esprit.

\section{Idées, idéaux et fictions de la raison}

Comme nous venons de le voir, l'interprétation complexe que donne Kant de la nature du sublime laisse ouverte la question de savoir si le sublime, en sa négativité, doit être interprété comme étant subjectif ou objectif. L'interprétation qu'en donne Kant peut en effet être comprise comme se préoccupant principalement de ces objets qu'ils soient réels ou fictifs - qui sont à l'origine de certains états d'" esprit " qui permettent à cette dernière de prendre conscience de la nature suprasensible de l'esprit. Mais elle peut être aussi comprise comme se préoccupant principalement de ces complexes sentiments de plaisir et de mécontentement quoccasionne la perpétuelle oscillation que subit la relation existant entre les diverses facultés.

Kant se refuse à toute interprétation deffinitive non parce qu'il se refuse à définir ce que peuvent ètre les attributs esthétiques des objets représentés - que ceux-ci soient réels, artificiels ou littéraires - ou des idées qui leur sont associées et qui, à travers la connaissance ou l'affectivité du sujet, permettent à celui-ci d'appréhender le sublime en tant que nature suprasensible de l'esprit mais parce qu'il préfère, comme le montre clairement les derniers fragments de l'Opus postumum, ne pas se prononcer sur la distinction implicite existant entre réalité et fiction tant que ne peut ètre résolu la question de savoir si le sublime doit être formule en terme d'objet ou d'état d' "esprit ".

La question qui, dès lors, se pose à nous est de savoir comment appréhender, à la lumière de ce que dit Kant du sublime, les thèmes centraux qui sont, au vingtième siècle, ceux de la poésie de la souffrance et, en miroir, réfléchir, avec plus de clarté, sur ce que peut vouloir signifier cette notion de sublime. Il me semble pour cela utile de voir en quoi le sublime kantien peut, à juste titre, ètre interprété en tant que fiction kantienne. Mais il est, pour cela, nécessaire d'examiner plus en détail ce qu'est exactement l'idée kantienne du sublime.

Ainsi Kant considère-t-il, de façon générale et comme le montre la Jäsche Logik, que les idées sont en relation avec la raison en tant que faculté de conclure. C'est ainsi qu'il écrit "la raison [...] consiste seulement dans la faculté de conclure, donc de juger médiatement ${ }^{37}$ ".

36. Ibid., p. $93 \ln (\mathrm{AK} \mathrm{V}, 177)$.

37. Kant, Opus postumum, op. cit., p. 198 (AK XXI, 11). 
Kant distingue néanmoins différents modes de la raison. Ainsi la raison théorico-pratique, pour Kant, "crée pour elle-mēme des objets, c'est-à-dire, des idées autonomes ${ }^{38}$ ". Ces idées sont de plus les représentations intuitives d'objets singuliers. "Ces idẻes, Kant écrit11, ne sont pas des concepts mais de pures intuitions : non des représentations discursives mais intuitives puisqu'il n'existe qu'un seul tel objet ${ }^{39}$ ".

La distinction faite ensuite entre raison théorico-spéculative d'une part et raison éthico-pratique d'autre part tire sa source de la philosophie transcendantale où la raison donne naissance à une émanation de l'idée. "La raison se crée elle-mème inévitablement des objets $^{40}$. "Le dessein de la philosophie transcendantale n'est pas de "fonder un objet, mais [d']établir a priori de façon exhaustive seulement les idées de ceux-ci [c'est-à-dire les objets] (en opposition avec les [objets de la philosophiel empirique[s] ${ }^{41}$ ".

De façon plus singulière encore, les deux modes, théorique et éthique, de la raison pratique se trouvent unis en un principe qui, écrit Kant, "lie les deux idées ${ }^{42}$ ". La raison technico-pratique est à l'origine de l'idée d'individualité (ce que Kant appelle conceptus singularis) du monde en tant que tel, en tant que tout (en tant que universum et non en tant que mundus) et la raison éthico-pratique à l'origine de l'idée de Dieu. Or, comme Kant l'écrit : " non seulement la raison technico-pratique, mais aussi la raison éthico-pratique se rencontrent dans l'idée : Dieu et le monde comme unité synthétique de la philosophie transcendantale ${ }^{43}$ ". Il reformulera la même idée, de façon légèrement différente, dans une note ajoutée ultérieurement: "le point suprême de la philosophie transcendante est ce qui réunit Dieu et le monde synthétiquement sous un principe ${ }^{44}$ ".

Ces deux formulations sont toutefois unifiées par le sujet rationnel qui, dans sa liberté, assure l'unité "de l'intuition de la nature dans l'espace et le temps ${ }^{45}$ "et le sentiment de spontanéité résultant d'une certaine forme de pensée systématique. "La raison, écrit Kant, fait cette unité transcendentale elle-mème ${ }^{46}$ ". C'est par ce mode de pensée, comme l'écrit Kant, "que nous nous faisons les objets mêmes par des connaissances synthétiques a priori à partir de concepts $^{47}$ "et que nous créons ces objets tout comme nous nous autocréons car c'est en créant ces objets que nous nous autocréons.

Mais de telles idées, d'après Kant, ne constituent pas pour la raison un agrégat mais un système "non de concepts objectifs mais d'idées subjectives ". La raison, de plus, constitue ce système d'idées subjectives "non hypothétiquement (problématiquement ou

38. Kant, Opus postumum, dans Kant's Gesammelte Schriften, herausgegeben von der Preußischen Akademie der Wissenschaften, op. cit., (AK XXI, 11).

39. Ibid., (AK XXI, 78-79).

40. Kant, Opus postumum, op. cit., p. 344, n. 445 (AK XXI, 83).

41. Ibid., p. 227 (AK XXI, 87-88).

42. Ibid., p. 197 (AK XXI, 11 ).

43. Ibid., p. 205-6 (AK XXI, 19).

44. Ibid., P. 210 (AK XXI, 23).

45. Kant, Opus postumum, dans Kant's Gesammelte Schriften, herausgegeben von der Preußischen Akademie der Wissenschaften, op. cit., (AK XXI, 22).

46. Ibid., (AK XXI, 23).

47. Kant, Opus postumum, op. cit. p. 208 (AK XXI, 21).

48. Ibid., p. 232. (AK XXI, 93). 
assertoriquement) mais apodictiquement ${ }^{49}$ ". La raison, en fin de compte, ne crée un tel système, de façon hypothétique, qu' en se produisant elle-mème $e^{50}$ ". C'est dans cette perspective que Kant conçoit finalement "la philosophie transcendantale [comme] faculté du sujet s'autodéterminant par le complexe systématique des idées qui font un problème de la détermination complète de celui-ci comme objet (l'existence de celui-ci) pour se constituer lui-même comme donné de l'intuition ". Ce que Kant résume par la formule "Tout comme se faire soi-même ${ }^{52}$ "qui conclut le paragraphe.

Kant abordant, dans l'Opus postumum, l'idée dans différents contextes, il n'est pas aisé de définir ce que signifie, pour lui, l'idée en tant que telle. Kant, cependant, utilise le concept de "fictions" dans un sens bien précis. Il écrit ainsi : "Les idées sont des principes subjectifs autocréés de la faculté de penser: pas des fictions mais des pensées ${ }^{53}$. "Il distingue cependant peu après les idées qui sont à la fois appréhendées par la perception ou la pensée et les simples pensées " peuvent ètre pensées mais non données à la perception (cogitabile, non dabile) ${ }^{54}$ ".

Kant insiste cependant sur le fait que "les idées ne sont pas des concepts mais de pures intuitions des représentations non discursives, mais intuitives ${ }^{55}$. Ainsi la raison peut-elle ètre, en philosophie transcendantale, dirigée de façon immédiate vers certaines idées qui possèdent une certaine autonomie " en tant que constituant un tout indépendant, par opposition à l'expérience et que la raison se constitue elle-méme par rapport à celui-ci en tant que système singulier ${ }^{56}$ ". Ces idées sont donc des pures intuitions qui ne sont ni de simples idées ni des idées proprement dites mais des idées transcendantales. Leurs objets ne sont rien d'autre que des "objets de la raison pure se constituant eux-mèmes en un système $[\ldots]^{57}$ ". Ces idées transcendantales sont au nombre de trois : Dieu, le monde et la personne (l'être humain "soumis au principe du devoir "). Ces idées transcendantales, comme l'écrit Kant, ne contribuent en rien au matériel mais seulement aux principes de la forme ${ }^{58}$ ". Kant explique ainsi, de façon plus explicite, que ces idées " ne peuvent rien apporter à la matière de la connaissance [...] mais seulement au principe de ce qui est formel ${ }^{59}$ "tout comme c'est le cas pour le principe de la liberté déterminé par l'impératif catégorique. Ce qui signifie que les idées transcendantales " précèdent les phénomènes de l'espace et le

49. Ibid, idem.

50. Ibid., idem

51. Ibid., P. 233 (AK XXI, 93).

52. Ibid., idem

53. Ibid., p. 217 (AK XXI, 29). La traduction de Marty utilise " affabulations " et non "fictions " mais fictions est le terme utilisé dans la traduction anglaise à laquelle se réfère l'article original (Note du traducteur).

54. Kant, Opus postumum dans Kant's Gesammelte Schriften, herausgegeben von der Preußischen Akademie der Wissenschaften, op. cit., (AK XX1, 30).

55. Kant, Opus postumum textes choisis et traduits, op. cit., p. 34 (AK XX1, 79).

56. Ibid., idem Traduction complétée par P. McCormick et L. Perrin.

57. Kant, Opus postumum, dans Kant's Gesammelte Schriften, herausgegeben von der Preußischen Akademie der Wissenschaften, op. cit., (AK XXI, 79).

58. Ibid., (AK XXI, 84).

59. Kant, Opus postumum, op. cit. p. 226 (AK XXI, 86-7). 
temps $^{60}$ ". Le système de ces idées transcendantales est la philosophie transcendantale $e^{61}$.

Les idées transcendantales ne peuvent donc ètre objectives. Elles sont au contraire subjectives dans la mesure où "la raison crée " de telles idées dans la faculté de penser du sujet en tant "qu'auteur de ses idées d'un point de vue théorique aussi bien que pratique ${ }^{62}$ ". Certaines choses de pensée se rapportent donc "particulièrement de façon privilégiée à des idées comme formes de la connaissance, par quoi l'objet se constitue lui-même comme être pensant ${ }^{63}$ ".

Et lorsque ces idées se rapportent à ce qu'il nomme un maximum - ce qui est le cas de Dieu, du monde et de la personne Kant leur donne alors le nom d'r idéal de la raison ". Les idéaux sont donc des idées qui représentent un maximum comme Kant l'explique en détails dans la section consacrée au sublime mathématique dans la Critique de la faculté de juger. Kant hésite cependant à affirmer que ces idées peuvent exister indépendamment de la pensée du sujet. Il déclare néanmoins qu'une idée au moins, "l'idée [...] d'une raison se constituant elle-même comme chose de pensée $e^{64}$ "[Kant précise plus loin qu'elle constitue un idéall, ne peut exister indépendamment de la pensée. Kant dit cependant des idéaux en tant qu'idées "que chacun d'eux [est] un maximum, et ils se rapportent à des choses qui sont hors de nous ${ }^{65}$ ". Les trois idées transcendantales mentionnées précédemment - Dieu, le monde, la personne - constituent donc de tels idéaux. Kant précise, dans un ajout tardif, que "les idées transcendantales sont différentes des idéaux ${ }^{66}$ ".

Kant semble donc ici suggérer que choses de pensée, simples idées, idéaux et idées transcendantales constituent une hiérarchie croissante. Toutes les idées ou tous les idéaux semblent être des choses de pensée mais seuls les idéaux semblent, d'une certaine manière, se rapporter "à des choses qui sont hors de nous "67 ". Ainsi les idéaux, en tant que pures représentations intuitives, semblent ètre le fruit du travail de la raison du sujet. De tels idéaux cependant, dont l'existence est dépendante de la pensée, se rapportent à des objets mentaux dont le contenu est, tout au moins en partie, relié à des objets qui ne sont pas purement mentaux.

Il nous faut cependant encore ici déterminer comment la doctrine kantienne du sublime négatif peut nous permettre de mieux comprendre et interpréter la poésie de la souffrance de Montale.

\section{Les fictions kantiennes}

Laissant pour un moment de cōté la nature de ces idées transcendantales que Kant appelle les idéaux de la raison afin de nous concentrer sur ce que dit Kant des idées proprement dites, nous trouvons, dans ses derniers écrits, une description particulièrement intéressante de ce que sont ces objets vers lesquels se tourne

60. Ibici. p. 227 (AK XXI, 88).

61. Ibid., p. 226-7 (AK XXI, 87).

62. Ibid., p. 232 (AK XXI, 93).

63. Ibid., p. 233 (AK XXI, 94).

64. Ibid., p. 215 (AK XXI, 27).

65. Ibid., p. 233 (AK XXI, 94).

66. (AK XXI, 81), trad. par P. McCormick et L. Perrin.

67. Ibid., p. 233 (AK XXI, 94). 
l'interprétation rationnelle du sublime. Kant, rapprochant de façon très intéressante sa pensée de celle de Spinoza sur les idées ${ }^{68}$, écrit : "Les idées sont des images [Bilder] (intuitions) créées a priori par la raison pure et qui, en tant que simples choses subjectives de la pensée et éléments de cette dernière, précèdent la connaissance des choses ${ }^{69}$ ". Kant souligne ici le sens particulier qu'il donne, à la fin de sa vie, de la raison créatrice. Il semble également faire ici une distinction, que nous avions anticipée, entre les choses de pensée qui sont purement subjectives et celles qui ne le sont pas.

Kant souligne de plus le caractère purement formel des idées en tant qu'images ou intuitions a priori. Les idées, d'après Kant, "sont les prototypes selon lesquels Spinoza pensait qu'on devait voir toutes choses en Dieu, suivant leurs formes : c'est-à-dire ce qui est formel dans les éléments à partir desquels nous faisons Dieu pour nousmêmes ${ }^{70}$ ". Kant semble donc accepter le fait que les idées puissent être interprétées comme étant des prototypes (ou archétypes) formels a priori mais il insiste cependant sur le fait que, selon sa doctrine qui veut que trois idées maximales soient des idéaux, même l'idée maximale de Dieu est entièrement dépendante de la faculté rationnelle du sujet conscient de soi.

Les réflexions de Kant sur les idées, telles que formulées dans l'Opus postumum, demeureraient cependant incomplètes si elles ne traitaient de la différence fondamentale qui existe, insiste Kant, entre une caractérisation négative et une caractérisation positive des idées. Et c'est dans le contexte de la caractérisation négative des idées que nous trouvons les plus intêressantes réflexions de Kant sur la problématique philosophique qui nous intéresse ici.

Kant tient à faire une distinction très claire entre les idées, d'une part, et ce qu'il appelle les "fictions", d'autre part. "Les idées ", résume-t-il dans un passage que nous avons déjà cité, "sont des principes subjectifs autocréés de la faculté de penser : pas des fictions mais des pensées ${ }^{71}$. Il s'empresse alors d'ajouter, probablement en guise d'exemple de ce qui est à la fois une idée et une

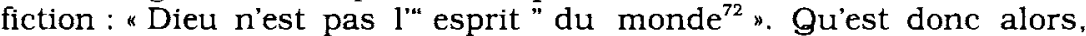
pour Kant dans l'Opus postumum, "une fiction "?

L'existence de certaines choses, pense Kant, peut ètre directement démontrée de façon empirique tandis que l'existence d'autres choses peut ètre démontrée a priori. L'existence de certaines choses a priori cependant ne peut ètre démontrée directement ni par un principe analytique ni par un principe synthétique du jugement. Il est néanmoins possible, croit Kant, de démontrer indirectement l'existence d'au moins une chose a priori : Dieu "quoique non donné objectivement ${ }^{73}$ "peut être, d'après Kant, pensé dans le domaine de la raison pratique régi par l'impératif catégorique de façon subjective et

68. Cf. Spinoza, Traité de la réforme de l'entendement, trad. par B. Rousset, Paris, Librairie philosophique J. Vrin, 1992.

69. Kant, Opus postumum, textes choisis et traduitt, op. cit,, p. 24 (AK XX1, 51). Trad. complétée par P. McCormick et L. Perrin.

70. Ibid., idem Traduction complétée par P. McCormick et L. Perrin.

71. Ibid., p. 217 (AK XXI, 29). Voir note 53.

72. Ibid, idem.

73. Ibid., p. 174 (AK XXII, 122). 
nécessaire car les devoirs humains peuvent ètre pensés en tant que " commandements divins ${ }^{74}$ ".

Il est essentiel, pour comprendre ce que Kant entend par fiction en tant qu'objet de l'interprétation rationnelle, de se reporter à la remarque où il déclare que, si l'on prétendait pouvoir directement démontrer a priori l'existence de quelque chose "comme une chose hypothétique au profit de phénomènes possibles ${ }^{75}$ ", on ne démontrerait en fait rien : on se contenterait d" imaginer ${ }^{76}$ ". Kant reprend ce terme un peu plus loin lorsqu'il aborde l'idée d'une "volontê morale et sainte commandant absolument ${ }^{77}$ " ou celle d'une "substance qui est unique dans son concept, et n'est pas soumise à une différence de classe venant de la raison humaine ${ }^{78}$. Un tel être, écrit Kant, est "pensé, ou plutôt imaginé, comme être de la nature ${ }^{79}$ ". Qu'est-ce donc que Kant entend ici par "imaginer " ?

Je propose ici, à la lumière de ce que Kant écrit, dans l'Opus postumum, sur la nature et les modes de la raison créatrice et de ses objets, de comprendre l' imagination " comme l'acte par lequel la raison produit, selon différents modes technico-pratiques (mais non éthico-pratiques) ces choses de pensée particulières que Kant oppose aux idées. L'imagination est la production créatrice de fictions par la raison. Une telle production se conduit, de façon caractéristique, sous forme de postulat hypothétique.

Kant utilise en de nombreuses occasions, dans l'Opus postumum, de tels postulats hypothétiques. Ainsi débute-t-il une longue réflexion sur l'autoposition pratique et l'idée de Dieu par un postulat hypothétique : "l'existence d'un tel être ne peut être postulée que dans une perspective pratique, à savoir la nécessité d'agir comme si je me trouvais sous cette conduite, redoutable, et en mème temps toujours salutaire, et porteuse en mème temps de garantie, dans la connaissance de tous mes devoirs en tant que commandements divins (tanquam non ceu) ; par suite, dans cette formule-ci, l'existence d'un tel être n'est postulée, ce qui serait aussi en soi contradictoire ${ }^{80}{ }^{*}$. Dieu, pour Kant, n'est pas une fiction.

L'idée pour Kant est ici de nous faire comprendre qu'une personne doit penser tous les devoirs comme des commandements divins $^{81}$. Le "comme si "doit ici, selon Kant, ètre interprété de façon relative et non de façon absolue afin de préserver la liberté humaine (il utilise ici tanquam [comme si, relativement] et non ceu [comme, absolument $\left.\right|^{82}$ ). Ainsi, pour Kant, les devoirs d'une personne sontils " comme des commandements divins ". Ce que Kant explicite par la suite de la manière suivante: "Les commandements sont divins (pracepta inviolabilia), c'est-à-dire ne permettent aucune atténuation,

74. Ibid., idem.

75. Ibid., p. 173 (AK XXII, 121).

76. Ibid., idem La traduction dit "inventer ", mais nous utilisons ici "imaginer " afin de préserver la concordance existant dans la traduction anglaise à laquelle se référe l'article original (Note du traducteur).

77. Ibid., p. 175 (AK XXII, 123).

78. Ibid., P. 175-6 (AK XXII, I23)

79. Ibid, p. 176 (AK XXII, 123).

80. Ibid, p. 170 (AK XXII, 116 ).

81. Ibid., p. 173 (AK XXII, 120).

82. Cf. la note de Kant: Ibid., p. 216 (AK XXI, 28).

83. Ibid., p. 205 (AK XXI, 17). 
et sur leur violation se produit le jugement de condamnation par la propre raison de l'homme, comme prononcé par une puissance morale, qui l'exécute ${ }^{84}$.

Ces postulats hypothẽtiques se présentent donc sous la forme d'analogies qui doivent être comprises de façon relative et non absolue. Ces analogies qui sous-tendent les postulats hypothétiques, formulées sur le mode relatif, implique dès lors une question de degré (Kant utilise ici " virtualiter " [virtualités] ${ }^{85}$ ).

Les postulats hypothétiques sont donc une forme d'assertion virtuelle dont le domaine de vérité relatif est également, pour ainsi dire, virtuel. Les postulats formulés dans de telles assertions virtuelles ne sont dès lors, en fait, que des "fictions "dans le sens où Kant l'entend lorsqu'il parle de ces " choses de pensée " particulières qui ne sont ni purement subjectives ni purement objectives mais, pour ainsi dire, virtuelles ou relatives.

Un des exemples les plus significatifs d'une telle fiction est celui du "réel ". "Le réel ", écrit Kant, "qui ne peut pas ètre un objet sensible et le réel qui doit l'être nécessairement, puisqu'il doit être un objet donné, ainsi comme espace et temps ne sont chacun qu'un ${ }^{86}$ ". La problématique particulière du lien existant entre fiction et nécessité qui se dévoile ici gagne à ètre lue à la lumière d'une des dernières remarques que fait Kant sur la nature de certaines idées dans le cadre de la philosophie transcendantale: "La philosophie transcendentale, écrit Kant, est la totalité absolue [du] système des idées qui appréhende de façon immédiate les objets (ens summum, summa intelligentia, etc.) qui, indépendamment de toute expérience, sont postulés par la raison pure comme objets [nécessaires] à la possibilité de [son] expérience ${ }^{87}$ ". Ce à quoi il ajoute, peu après : "La philosophie transcendantale porte ce nom parce qu'elle précède la métaphysique et fournit à celle-ci ses principes ${ }^{88}$ ". Pouvons-nous dès lors conclure, à la lumière de ces réflexions sur les idées, les idéaux et la fiction, que, parmi les différents principes de la philosophie transcendantale, il en est un particulier qui, virtuel, constitue ce qu'on appelle la fiction de la raison?

Cela reviendrait à dire que l'objet d'une interprétation rationnelle de l'art que constitue, par exemple, Maison sur la mer de Montale, parce qu'il peut évoquer en nous l'expérience du sublime, n'est un objet que de façon relative et que de plus, comme tout autre objet virtuel tel que "le réel ", il n'est, étant à la fois objectif et subjectif, qu'une fiction au sens kantien du terme.

Revenons maintenant un instant, avant de conclure, à l'un des poèmes d'Os de seiche afin de nous rappeler comment un tel poème peut, aujourd'hui encore, nous inviter à réfléchir sur le lien qui peut exister entre poésie et vécu en des temps de troubles historiques, culturels et sociaux :

84. Ibid., p. 205 (AK XXI, 20).

85. Ibid., p. 197 (AK XXI, 11).

86. Ibid., p. 192 (AK XXII, 59).

87. Kant, Opus postumum, dans Kant's Gesammelte Schriften, herausgegeben von der Preußischen Akademie der Wissenschaften, op. cit., (AK, XXI, 80).

88. Ibid., (AK XXI, 81). 
Guelquefois descendant

les arides talus désormais

exilés de l'automne

qui les gonflait, riche de sève,

je n'avais plus au cœur la roue

des saisons et le suintement

du temps inexorable ;

mais c'était le pressentiment

de toi qui emplissait mon âme,

tout surpris dans le halètement

de l'air auparavant inerte

sur les roches au bord du chemin.

Oui, je m'en avisais, la pierre

se voulait arracher, tendue toute

vers un embranchement invisible :

la dure matière pressentait

le gouffre proche, palpitait :

et les touffes de roseaux avides

disaient aux eaux cachées,

en s'agitant, leur consentement.

Tu rachetais, ample ètendue,

jusqu'à la souffrance des pierres :

pour ton allégresse était juste

l'immobilité du finj ${ }^{89}$.

Je ne sais pas s'il est possible d'interpréter de façon critique le jeu d'ombre et de lumière automnale qui éclaire ici le sublime dans ses aspects objectifs et subjectifs. N'est-il, après tout, autre chose qu'une réminiscence de la Ligurie où Montale passa sa jeunesse ? Et si tel est le cas, comment pourrions-nous faire nôtre un tel poème ? Mais en cette époque qui se veut postmoderne et, surtout, à la fin du siècle le plus sanglant de notre histoire - Nuit et Brouillard inexorables - une telle question a-t-elle encore une quelconque pertinence ?

Je crois seulement pouvoir répondre que nous avons trop tendance à négliger, en ces temps de bouleversements historiques, culturels et nationaux, notre vie ainsi que celles des sociétés qui nous entourent de même que, et sans raison véritable si ce n'est par pédanterie ou snobisme philosophiques, celle de l'idée et de l'idéal critique d'une pensée interprétative, d'une pensée critique qui pourrait nous permettre d'établir un lien entre la poésie et le vécu et nous donner ainsi les moyens de créer un nouveau langage critique fondé sur la résonnance personnelle, qu'elle soit individuelle ou sociale. Et ce sont peut-être certaines de ces ressources critiques, philosophiques - et peut-être mème métaphysiques - qui se dévoilent à nos yeux ou résonnent à nos oreilles, certes de façon abstraite, dans les poèmes de Montale, ou dans tout autre poème de la souffrance, en

89. Extrait de Quelquefois descendant d'Eugenio Montale, Os de seiche, traduit par Patrice Angelini avec le concours de Louise Herlin et Georges Brazzola, Paris, Gallimard, 1966, p. 119. 
cette fin de vingtième siècle. Car, même en cette époque postmoderne, rien ne saurait nous interdire de méditer sur l'influence bien réelle, à la fois objective et subjective - puisque fictive - qu'ils ont sur nos vies.

Département de philosophie

Université d'Ottawa

Traduit de l'anglais par Lionel Perrin 